\title{
Kapasitif Açısal Pozisyon Sensörü için CORDIC ile Arktanjant Fonksiyonu Uygulanması
}

\author{
Muhammet Rojhat Kara ${ }^{1}$, Emrehan Yavsan ${ }^{2 *}$, Mehmet Karalı ${ }^{2}$, Mehmet Akif Erişmiş ${ }^{3}$ \\ ${ }^{1}$ Karamanoğlu Mehmetbey Üniversitesi, Mühendislik Fakültesi, Elektrik-Elektronik Mühendisliği Bölümü, Karaman, Türkiye (ORCID: 0000-0002-0353-1902) \\ ${ }^{2}$ Necmettin Erbakan Üniversitesi, Mühendislik ve Mimarlık Fakültesi, Mekatronik Mühendisliği Bölümü, Konya, Türkiye (ORCID: 0000-0001-9521-4500) \\ ${ }^{3}$ Necmettin Erbakan Üniversitesi, Mühendislik ve Mimarlık Fakültesi, Elektrik-Elektronik Mühendisliği Bölümü, Konya, Türkiye (ORCID: 0000-0001-6837-2087)
}

( $1^{\text {st }}$ International Conference on Computer, Electrical and Electronic Sciences ICCEES 2020 - 8-10 Ekim 2020)

(DOI: 10.31590/ejosat.802008)

ATIF/REFERENCE: Kara, M.R., Yavşan, E., Karalı, M. \& Erişmiş, M.A. (2020). Kapasitif Açısal Pozisyon Sensörü için CORDIC ile Arktanjant Fonksiyonu Uygulanması. Avrupa Bilim ve Teknoloji Dergisi, (Özel Sayı), 160-164.

\section{$\ddot{O} z$}

Arktanjant fonksiyonu güç sistemlerinde, AC devrelerdeki analizlerde, enkoderlerde açı ve hız hesabında sistemlerin izlenmesi ve kontrolü için vazgeçilmez bir parçadır. $\mathrm{Bu}$ tür kontrol uygulamaları çoğunlukla mikrodenetleyicilerde yapılmaktadır fakat mikrodenetleyicilerin işlem kapasiteleri kısıtlıdır. Arktanjant fonksiyonu bir mikrodenetleyici içerisinde seri açılımı yöntemi ile hesaplanmaya çalışılırsa hem çok fazla işlem gücü gerekir hem de uzun zaman harcar. Ayrıca seri açılımı yönteminde hassasiyetin artması için daha fazla terim gerekir ve bu işlem yoğunluğunu daha da arttırır. Fakat arama tabloları ve CORDIC algoritması kullanılarak bu işlemler hem daha hızlı hem de bit düzeyinde kaydırma, toplama ve çıkarma gibi basit işlemlerle yapılabilmektedir. $\mathrm{Bu}$ gibi bit düzeyindeki işlemler ortalama bir mikrodenetleyicinin rahatça yapabileceği işlerdir. Bu çalı̧̧mada kapasitif rotary enkoderlerde rotor açısı ve hız hesabı için CORDIC algoritması kullanılmış ve bu işlemler ARM çekirdeğini kullanan bir mikrodenetleyicide gerçekleştirilmiştir. Sonuçlar hazır matematik kütüphanelerindeki arktanjant fonksiyonu ile kıyaslandığında CORDIC algoritmasının radyan cinsinden sonuç için $10^{-6}$ ' lık bir hassasiyet ile aynı işlemleri atan 2 fonksiyonundan yaklaşık 9 kat ve $\operatorname{atan} 2 f$ fonksiyonundan 2 kat daha hızlı yaptı̆̆ gözlemlenmiştir.

\section{Arctangent Function Implementation with CORDIC for a Capacitive Angular Position Sensor}

\begin{abstract}
Arctangent function is an indispensable part for monitoring and control of systems in power systems, analysis of AC circuits, angle and speed calculation in encoders. Such control applications are mostly done in microcontrollers, but the processing capacity of microcontrollers are limited. If the arctangent function is tried to be calculated by serial expansion method in a microcontroller, it requires both a lot of CPU power and a long time. In addition, more terms are required to increase the accuracy in the series expansion method, and this further increases the density of the process. However, using the look up tables and the CORDIC algorithm, these operations can be done both faster and with simple operations such as bit shift, addition and subtraction. These operations are quite doable even for an average microcontroller. In this study, CORDIC algorithm was used for the rotor angle and speed calculation in capacitive rotary encoders and these operations were performed in a micro controller that uses ARM core. When the results are compared with the arctangent function in the math.h library, it has been observed that the CORDIC algorithm performs the same
\end{abstract}

\footnotetext{
* Sorumlu Yazar: Necmettin Erbakan Üniversitesi, Mühendislik ve Mimarlık Fakültesi, Mekatronik Mühendisliği Bölümü, Konya, Türkiye, ORCID: 0000-0001-9521-4500, eyavsan@erbakan.edu.tr
} 
operations approximately 9 times faster than atan 2 function and 2 times faster than atan $2 f$ fuction with a precision of $10^{-6}$ in radians.

Keywords: Arctangent, CORDIC, Capacitive Sensor, Microcontroller, Position Sensor.

\section{Giriş}

Koordinat döndüren sayısal bilgisayar (CORDIC) aritmetiğinin ana konsepti, iki boyutlu geometrinin basit ve antik kurallarını temel almaktadır. Fakat özellikle trigonometrik fonksiyonların hesabında kullanılan bu algoritmanın uygulama için iteratif olarak yazılması ilk olarak 1959 yılında Jack E. Volter [1] tarafından gerçekleştirilmiştir.

İlerleyen dönemde Cochran [2] CORDIC algoritmasını çeşitli algoritmalarla kıyaslayarak özellikle bilimsel bir hesap makinesi işlemleri için daha iyi bir seçenek olduğunu göstermiştir. CORDIC algoritmasının popüleritesi ve kullanımı daha sonralarında trigonometrik, logaritmik fonksiyon hesaplarında, karmaşık sayı çarpımlarında, sinyal işleme için tekil değer ayrışımda, görüntü işlemede ve bu gibi çeşitli uygulamalardaki etkinliği görülüp başarılı sonuçlar alındıkça giderek artmıştır. Günümüzde de halen aktif kullanılmakta olan etkin bir algoritmadır.

Yüksek çözünürlük ve doğruluklu açısal pozisyon sensörleri genellikle motor pozisyon kontrolünde geri bildirim olarak kullanılmaktadır. Kapasitif sensörler temassız ölçüm, yüksek ölçüm hassasiyeti, basit yap1, düşük maliyet ve zorlu ortamlarda çalışabilme özellikleri ile öne çıkmıştır [3]. Yapılan çalışmalarda kullanılan kapasitif açısal pozisyon sensörlerinin yapısı gereği çıkış sinyalleri sinus ve kosinüs sinyalleri şeklinde oluşmaktadır. Bu sinyallerin sensörün rotor açısı ile bağdaştırılabilmesi için arktanjant işlemi kullanılmaktadır. CORDIC algoritması bu lineer olmayan fonksiyonu hesaplarken kullanıma konulmuştur.

Zheng ve arkadaşları geliştirdikleri kapasitif enkoderin açı hesabında arktanjant fonksiyon sonucu hesaplamak için CORDIC algoritmasını tercih etmişlerdir [4]. Burada CORDIC algoritmasını paralel işlem yapan bir FPGA (alanda programlanabilir kapı dizisi) üzerinde uygulamışlardır.

$\mathrm{Bu}$ çalışmada diğer çalışmalardan farklı bir tasarımda olan kapasitif bir açısal pozisyon sensörü için arktanjant hesabında CORDIC algoritması uygulanmıştır. Diğer çalışmalarda kullanılan paralel işlem yapabilen bir birimden farklı olarak seri işlem yapan ARM tabanlı bir mikrodenetleyici kullanılmıştır. Ayrıca sensör çıkışındaki non-lineeritenin sebebi aranırken diğer araştırmacılar tarafından genellikle mekanik yapıdan kaynaklandığı vurgusunun yanında, ki hatalar çoğunlukla bundan kaynaklanmaktadır, arktanjant işleminin de bir non-lineerite getirdiği fark edilmiştir. CORDIC algoritması çıkışındaki non-lineerite ile bu fonksiyonlar kıyaslanmıştır. Böylece kapasitif sensörlerdeki uygulama maliyeti azaltılırken sensör performansının arttırılması sağlanmıştır.

\section{Materyal ve Metot}

\subsection{Kapasitif Açısal Pozisyon Sensörü}

Kapasitif açısal pozisyon sensörleri basit bir kapasitörün oluşturulma mantığına dayanıp Denklem (1)'deki parametrelerden birinin kontrollü bir şekilde değiştirilmesiyle elde edilebilirler.

$$
C=\varepsilon \frac{A}{d}
$$

bu denklemde $C$ plakar arasında oluşan kapasitansı, $\varepsilon$ dielektrik katsayısını, $A$ plakaların birbirlerine bakan yüzey alanlarını ve $d$ plakalar arası mesafeyi temsil eder. İletkenlerin kesişen alan değişimine bağlı kapasitans değişimine bir örnek olarak Şekil 1 ve 2 deki kapasitif sensörler gösterilebilir [5]. Bu kapasitif sensörlerin mekanik yapılarına ve girişlerine verilen sinyallerine bağlı olarak çıkışlarında sinüs ve kosinüs sinyalleri elde edilir. Bu sinyaller sensör açısının lineer bir grafiğe bağlı olarak elde edilmesini sağlayan arktanjant fonksiyonu hesaplanmasında girdi olarak kullanılır.

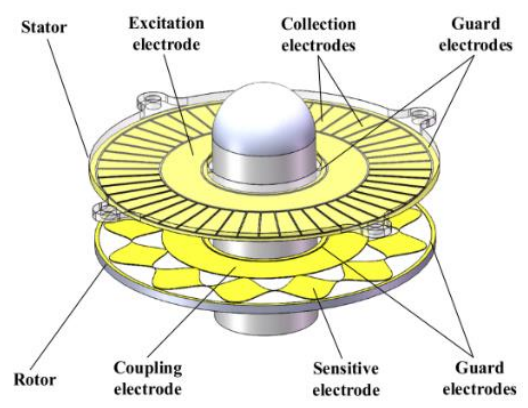

Şekil 1. Kapasitif sensör mekaniği [5] 


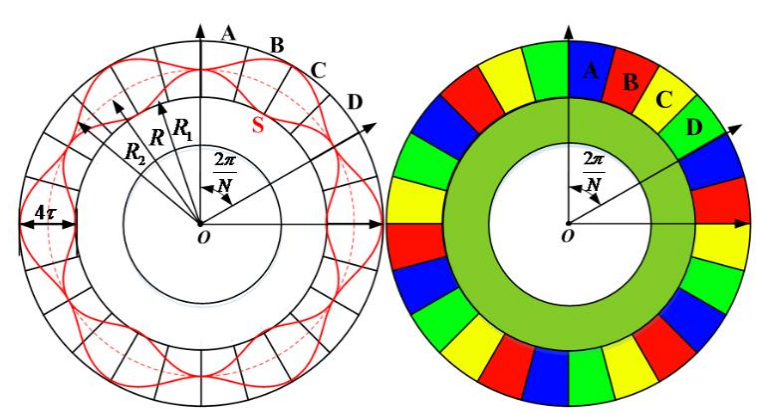

Şekil 2. Kapasitif açı sensörü rotor ve statoru [5]

Arktanjant fonksiyonu özellikle de düşük maliyetle çözülmesi gerek mikrodenetleyici sistemlerinde, mikrodenetleyicilerde FPU (floating point unit) olmadığı için hesaplaması zor bir fonksiyondur. Bu gibi durumlara çözüm olarak CORDIC algoritması kullanılarak sadece toplama, çıkarma ve bit kaydırma ile arktanjant işlemi yapılabilir.

\subsection{CORDIC Algoritması}

CORDIC algoritması sadece toplama, çıkarma ve bit kaydırma operatörlerini kullanarak iteratif bir şekilde lineer olmayan fonksiyonların hesaplanamasında kullanılmaktadır. CORDIC algoritması Şekil 3’te gösterildiği gibi $\left(X_{i}, Y_{i}\right)$ vektörünün $\theta$ açısı kadar döndürülmesi prensibine dayanır. Döndürme işlemi denklem 2 ve 3 ’teki gibi temsil edilmişsir [6].

$$
\begin{gathered}
X_{j}=R \cos (\theta+\beta)=X_{i} \cos (\theta)-Y_{i} \sin (\theta) \\
Y_{j}=R \sin (\theta+\beta)=X_{i} \sin (\theta)-Y_{i} \cos (\theta)
\end{gathered}
$$

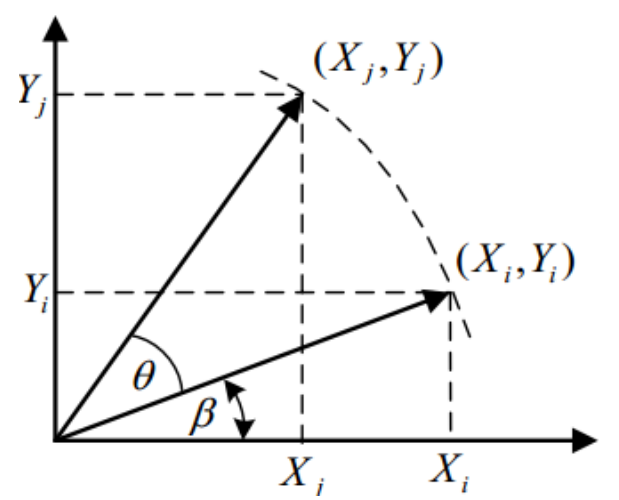

Şekil 3. CORDIC algoritması temel mantı̆̆ [4]

Eğer denklem yeniden düzenlenirse:

$$
\begin{gathered}
X_{j}=\cos (\theta)\left[X_{i}-Y_{i} \tan (\theta)\right], \\
Y_{j}=\cos (\theta)\left[X_{i} \tan (\theta)+Y_{i}\right] .
\end{gathered}
$$

Dönüş açıları $\theta_{n}=\delta_{n} \arctan \left(2^{-n}\right)$ şeklinde yazılabilir. Burada $\delta_{n}$ her eleman için dönüş yönünü belirlemektedir ve böylece tanjant ile çarpma terimi basit bir bit kaydırma işlemine dönüştürülmüş olur. Herhangi bir açı hesabı için denklemler Denklem (6) ve (7)'deki gibi iterative hale getirilmiştir.

$$
\begin{aligned}
& X_{n+1}=\cos \theta_{n}\left[X_{n}-\delta_{n} 2^{-n} Y_{n}\right], \\
& Y_{n+1}=\cos \theta_{n}\left[\delta_{n} 2^{-n} X_{n}+Y_{n}\right] .
\end{aligned}
$$

İterasyon sayısı sonsuza giderken $\cos (\theta)$ terimi 0.607253 değerine oturmaktadır. $\cos (\theta)$ 'nın oturma değerinden yola çıkılarak son iteratif ifadeler için Denklem (8), (9) ve (10) kullanılmıştır.

$$
\begin{gathered}
X_{n+1}=X_{n}-\delta_{n} 2^{-n} Y_{n}, \\
Y_{n+1}=\delta_{n} 2^{-n} X_{n}+X_{n}, \\
Z_{n+1}=Z_{n}-\delta_{n} \theta_{n} .
\end{gathered}
$$

$\delta_{n}$ dönüş yönüne göre \pm 1 alırken $Z_{n}$ ifadesi her iterasyonda yapılan açıyı tutup toplamaktadır. $Y_{n}$ ifadesi pozitifken $\delta_{n}=-1$, negatifken $\delta_{n}=+1$ değerini almaktadır. $Y_{n}$ ifadesi 0 olduğunda algoritma sonlanmakta ve $Z_{n}$ içerisinde tutulan $\operatorname{sayı} \arctan \left(\frac{Y_{0}}{X_{0}}\right)$ olmaktadir. 


\subsection{Uygulama Çıktıları}

CORDIC algoritması STM32F103C8T6 mikrodenetleyicisinde herhangi bir hazır kütüphane kullanılmadan yazılmış ve math.h kütüphanesi içindeki atan2 ve atan $2 f$ fonksiyonları ile kıyaslanmıştır. Aşağıdaki şekillerde bu hazır fonksiyonlarla CORDIC algoritmasının işlem süreleri verilmiştir. atan 2 ve atan $2 f$ hazır fonksiyonların çalışma süreleri yaklaşık olarak sırasıyla 3.4 ms ve $700 \mu$ s ölçülürken CORDIC algoritmasının çalışma süresi yaklaşık $380 \mu$ s ölçülmüştür.

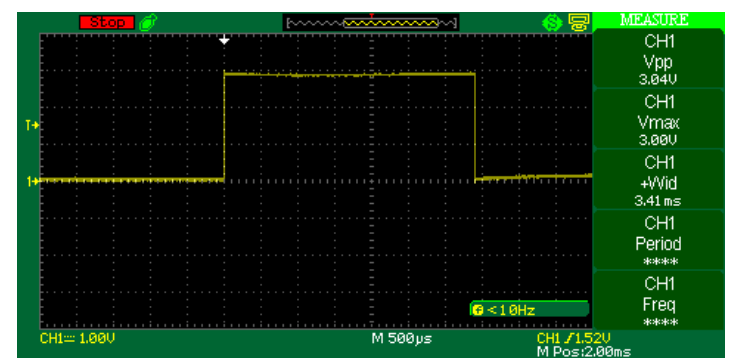

Şekil 4. Atan2 fonksiyonun yaklaşık çalışma hızı $3.4 \mathrm{~ms}$

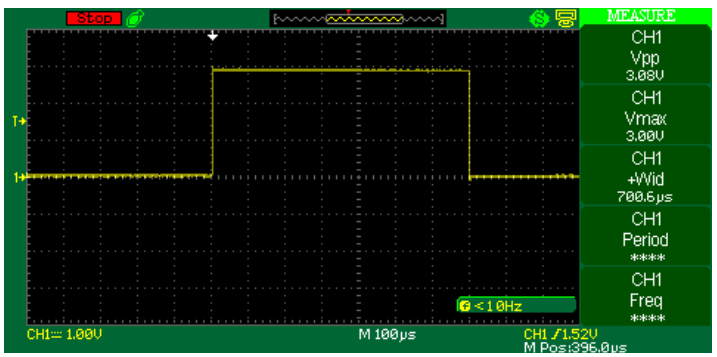

Şekil 5. Atan2f fonksiyonun yaklaşık çalışma hızı $700 \mu \mathrm{s}$

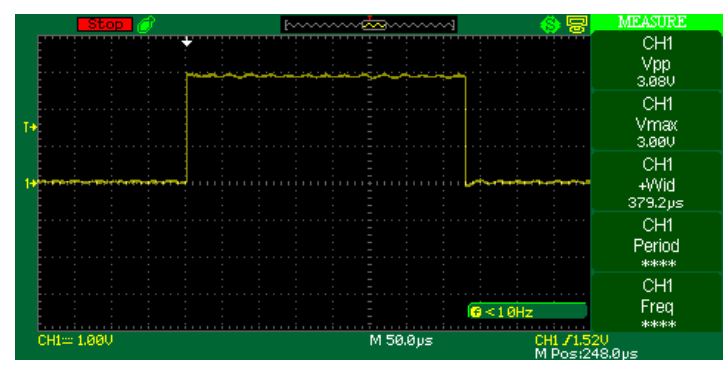

Şekil 6. CORDIC algoritmasının yaklaşık çalışma hızı yaklaşık $380 \mu \mathrm{s}$

Şekil 4, 5 ve 6'da gösterilen kıyaslama sonucunda CORDIC algoritmasının atan2 fonksiyonundan yaklaşık 9 kat atan $2 f$ fonksiyonundan ise yaklaşık 2 kat hızlı çalıştığ gösterilmiştir. Bununla beraber CORDIC algoritmasının non-lineerite hatası hesaplanmış ve diğer fonksiyonlardan daha düşük olduğu Şekil 7'de verilmiştir.

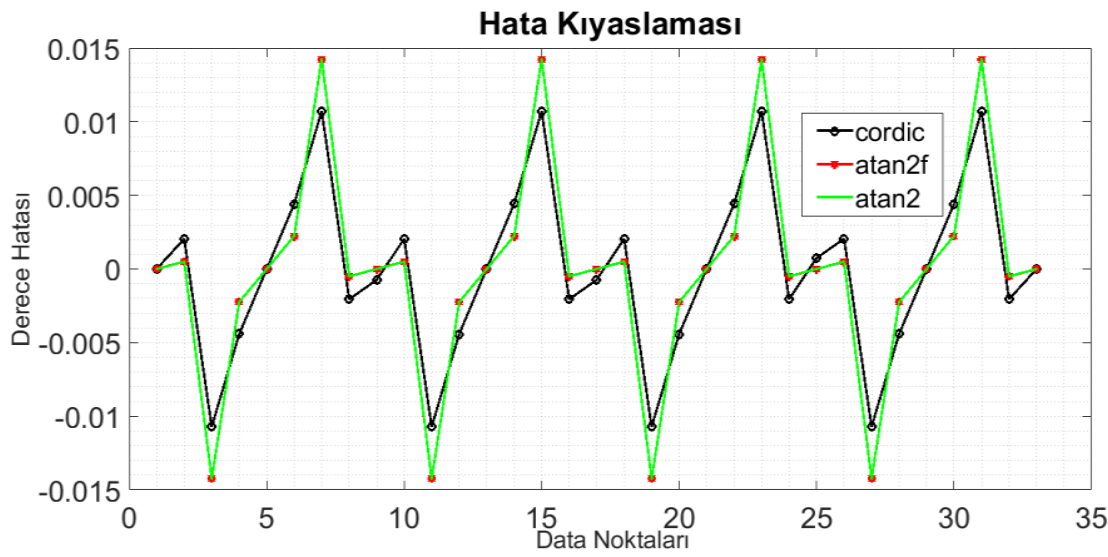

Şekil 7. Non-lineerite hata kıyaslaması 
Açı hatalarının karelerinin ortalamalarının karekökü (RMSE) ise Tablo 1'de verilmiştir. Burada da CORDIC atan2 ve atan $2 f$ fonksiyonlarına göre daha iyi bir performans göstermiştir.

Tablo 1. Açı kare ortalamalarının karekökü (rmse)

\begin{tabular}{|c|c|c|}
\hline CORDIC & ATAN2 & ATAN2F \\
\hline 0.0058010 & 0.0071082 & 0.0071087 \\
\hline
\end{tabular}

\section{Sonuç}

Bu çalışmada özgün tasarımlı kapasitif bir açısal pozisyon sensöründe açı hesabı için kullanılan arktanjant fonksiyonu CORDIC algoritması ile gerçekleştirilmiştir. Gerçekleştirilen CORDIC algoritmasının çalışma hızı hazır kütüphane fonksiyonlarıyla kıyaslanmıştır. CORDIC algoritmasının diğer hazır fonksiyonlardan daha hızlı çözüm ürettiği görülmüştür.

Kapasitif rotary enkoderlerdeki hataların çoğu algılayıcı mekaniğindeki uyumsuzluklardan veya üretim hatalarından kaynaklanmaktadır. Bu çalışmayla kapasitif enkoderler veya benzer algılayıcılardaki hataların mekanik kaynaklı olmasının yanında elektronik taraftaki doğrusal olmayan işlemlerin de hata getirebileceği gösterilmiştir. Bu çalışmada mekanik kaynaklı olmayan bu hatalar, CORDIC algoritmasıyla daha düşük seviyelere çekilmiştir.

CORDIC algoritması FPU donanımın bulunmadığı temel mikroişlemcilerle gerçekleştirilen uygulamalar için hız ve düşük hata oranın yanında düşük maliyet için de değerlendirilmesi gereken ciddi bir alternatiftir.

\section{Teşekkür}

Bu çalışma "Kapasitif Rotary Enkoderler için Analog Anahtarlama Tabanlı Okuma Devresi” başlıklı TÜBİTAK tarafından 2210D kapsamında 1649B021908049 burs başvuru numarasıyla desteklenen yüksek lisans tez çalışmasından bir bölümün derlenip detaylandırılmasıyla hazırlanmıştır.

\section{Kaynakça}

[1] J. E. Volder, "The CORDIC trigonometric computing technique," IRE Trans. Electron. Computers, vol. EC-8, pp. 330-334, Sept. 1959.

[2] D. S. Cochran, “Algorithms and accuracy in the HP-35," HewlettPackard J., pp. 1-11, Jun. 1972.G. Brasseur, "Design Rules for Robust Capacitive Sensors," IEEE Trans.

[3] D. Zheng, S. Zhang, S. Wang, C. Hu and X. Zhao, "A Capacitive Rotary Encoder Based on Quadrature Modulation and Demodulation," in IEEE Transactions on Instrumentation and Measurement, vol. 64, no. 1, pp. 143-153, Jan. 2015.

[4] D. Zheng, S. Zhang, Y. Zhang, and C. Fan, "Application of CORDIC in capacitive rotary encoder signal demodulation," in Proc. 8th IEEE Instrum. Control Technol. (ISICT), Jul. 2012, pp. 61-65.

[5] B. Hou, Z. Tian, C. Li, Q. Wei, B. Zhou and R. Zhang, "A capacitive rotary encoder with a novel sensitive electrode," 2017 IEEE SENSORS, Glasgow, 2017, pp. 1-3, doi: 10.1109/ICSENS.2017.8234143.

[6] S. Ravichandran and V. Asari, "Implementation of unidirectional CORDIC algorithm using precomputed rotation bits," The 2002 45th Midwest Symposium on Circuits and Systems, 2002. MWSCAS-2002., Tulsa, OK, USA, 2002, pp. III-III. 